\title{
A Focused Investigation of Expedited, Stack of Three Shocks Versus Chest Compressions First Followed by Single Shocks for Monitored Ventricular Fibrillation/Ventricular Tachycardia Cardiopulmonary Arrest in an In-hospital Setting
}

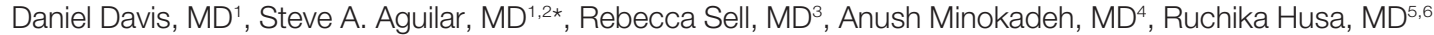

\begin{abstract}
${ }^{1}$ Department of Emergency Medicine, University of California San Diego, San Diego, California; '2Department of Emergency Medicine, Kaiser Permanente Medical Center, San Diego, California; ${ }^{3}$ Division of Pulmonary Critical Care, University of California San Diego, San Diego, California; ${ }^{4}$ Department of Anesthesiology, University of California San Diego, San Diego, California; ${ }^{5}$ Division of Cardiology, University of California San Diego, San Diego, California; ${ }^{6}$ Division of Cardiology, The Ohio State University, Columbus, Ohio.
\end{abstract}

BACKGROUND: In cases of in-hospital-witnessed ventricular fibrillation/ventricular tachycardia (VF/VT) arrest, it is unclear whether cardiopulmonary resuscitation prior to defibrillation attempt or expedited stacked defibrillation attempt is superior.

METHODS: Retrospective, observational study of all admitted patients with continuous cardiac monitoring who suffered VF/VT arrest between July 2005 and June 2013. In the stacked shock period (2005-2008), institutional protocols advocated early defibrillation with administration of 3 stacked shocks with brief pauses between each single defibrillation attempt to confirm sustained VF/VT. During the initial chest compression period (2008-2011), the protocol was modified to perform a 2-minute period of chest compressions prior to each defibrillation, including the initial. In the modified stack shock period (2011-2013), for a monitored arrest, defibrillation attempts were expedited with up to 3 successive shocks administered for persistent VF/VT. In unmonitored arrest, chest compressions and ventilations were initiated prior to defibrillation. The primary outcome measure was survival to hospital discharge.

RESULTS: Six hundred sixty-one cardiopulmonary arrests were recorded during the study period, with 106 patients (16\%) representing primary VF/VT. The incidence of VF/NT arrest did not vary significantly between the study periods $(P=0.16)$ Survival to hospital discharge for all primary VF/ VT arrest victims decreased, then increased significantly from the stacked shock period to initial chest compression period to modified stacked shock period $(58 \%, 18 \%, 71 \%$, respectively, $P<0.01)$. Specific group differences were significant between the initial chest compression versus the stacked and modified stacked shock groups (all $P<0.01$ ).

CONCLUSION: Data suggest that monitored VF/NT should undergo expeditious defibrillation with use of stacked shocks. Journal of Hospital Medicine 2016;11:264-268. (C) 2015 Society of Hospital Medicine
Cardiopulmonary arrest (CPA) is a major contributor to overall mortality in both the in- and out-of-hospital setting. ${ }^{1-3}$ Despite advances in the field of resuscitation science, mortality from CPA remains high. ${ }^{1,4}$ Unlike the out-of-hospital environment, inpatient CPA is unique, as trained healthcare providers are the primary responders with a range of expertise available throughout the duration of arrest.

There are inherent opportunities of in-hospital cardiac arrest that exist, such as the opportunity for near immediate arrest detection, rapid initiation of highquality chest compressions, and early defibrillation if indicated. Given the association between improved rates of successful defibrillation and high-quality chest

*Address for correspondence and reprint requests: Steve A. Aguilar, MD, UCSD Center for Resuscitation Science, Department of Emergency Medicine, 200 W. Arbor Dr. \#8676, San Diego, CA 92103; Telephone: 619-528-5164; Fax: 619-528-6024; E-mail: steveaguilarmd@gmail.com

Additional Supporting Information may be found in the online version of this article.

Received: June 19, 2015; Revised: September 18, 2015; Accepted: September 27, 2015

2015 Society of Hospital Medicine DOI 10.1002/jhm.2499

Published online in Wiley Online Library (Wileyonlinelibrary.com). compressions, the 2005 American Heart Association (AHA) updates changed the recommended guideline ventricular fibrillation/ventricular tachycardia (VF/VT) defibrillation sequence from 3 stacked shocks to a single shock followed by 2 minutes of chest compressions between defibrillation attempts. ${ }^{5,6}$ However, the recommendations were directed primarily at cases of outof-hospital VF/VT CPA, and it currently remains unclear as to whether this strategy offers any advantage to patients who suffer an in-hospital VF/VT arrest. $^{7}$

Despite the aforementioned findings regarding the benefit of high-quality chest compressions, there is a paucity of evidence in the medical literature to support whether delivering a period of chest compressions before defibrillation attempt, including initial shock and shock sequence, translate to improved outcomes. With the exception of the statement recommending early defibrillation in case of in-hospital arrest, there are no formal AHA consensus recommendations. ${ }^{5,8,9}$ Here we document our experience using the approach of expedited stacked defibrillation shocks in persons experiencing monitored in-hospital VF/VT arrest. 


\begin{tabular}{|c|c|c|c|}
\hline Protocol Variable & $\begin{array}{l}\text { Stack Shock Period } \\
\quad(2005-2008)\end{array}$ & $\begin{array}{l}\text { Initial Chest Compression } \\
\text { Period (2008-2011) }\end{array}$ & $\begin{array}{l}\text { Modified Stack Shock } \\
\text { Period (2011-2013) }\end{array}$ \\
\hline Defibrillator type & Medtronic/Physio Control LifePak 12 & Zoll E Series & Zoll E Series \\
\hline Joule increment with defibrillation & 200J-300J-360J, manual escalation & 120J-150J-200J, manual escalation & 120J-150J-200J, automatic escalation \\
\hline $\begin{array}{l}\text { Distinction between monitored and } \\
\text { unmonitored in-hospital cardiopulmonary } \\
\text { arrest }\end{array}$ & No & Yes & Yes \\
\hline Chest compressions prior to initial defibrillation & No & Yes & $\mathrm{No}^{*}$ \\
\hline Initial defibrillation strategy & $\begin{array}{l}3 \text { expedited stacked shocks with a brief } \\
\text { pause between each single defibrillation } \\
\text { attempt to confirm sustained VFNT }\end{array}$ & $\begin{array}{l}2 \text { minutes of chest compressions prior to initial } \\
\text { and in between attempts }\end{array}$ & $\begin{array}{c}3 \text { expedited stacked shocks with a brief pause } \\
\text { between each single defibrillation attempt to } \\
\text { confirm sustained VFNT }\end{array}$ \\
\hline Chest compression to ventilation ratio & $15: 1$ & $\begin{array}{l}\text { Continuous chest compressions with ventilation } \\
\text { at ratio 10:1 }\end{array}$ & $\begin{array}{l}\text { Continuous chest compressions with ventilation at } \\
\text { ratio 10:1 }\end{array}$ \\
\hline Vasopressors & Epinephrine $1 \mathrm{mg}$ IV/10 every 3-5 minutes. & $\begin{array}{l}\text { Epinephrine } 1 \mathrm{mg} \text { IV/10 or vasopressin } \\
40 \text { units IV/10 every 3-5 minutes }\end{array}$ & $\begin{array}{l}\text { Epinephrine } 1 \mathrm{mg} \text { IV/10 or vasopressin } \\
40 \text { units IV/10 every } 3-5 \text { minutes. }\end{array}$ \\
\hline
\end{tabular}

NOTE: Abbreviations: IO, intraosseous; IV, intravenous; VF, ventricular fibrillation; VT, ventricular tachycardia. *Only if monitored or witnessed at time of arrest.

\section{METHODS}

\section{Design}

This was a retrospective study of observational data from our in-hospital resuscitation database. Waiver of informed consent was granted by our institutional investigational review board.

\section{Setting}

This study was performed in the University of California San Diego Healthcare System, which includes 2 urban academic hospitals, with a combined total of approximately 500 beds. A designated team is activated in response to code blue requests and includes: code registered nurse $(\mathrm{RN})$, code doctor of medicine (MD), airway MD, respiratory therapist, pharmacist, house nursing supervisor, primary $\mathrm{RN}$, and unit charge RN. Crash carts with defibrillators (ZOLL R and E series; ZOLL Medical Corp., Chelmsford, MA) are located on each inpatient unit. Defibrillator features include real-time cardiopulmonary resuscitation (CPR) feedback, filtered electrocardiography (ECG), and continuous waveform capnography.

Resuscitation training is provided for all hospital providers as part of the novel Advanced Resuscitation Training (ART) program, which was initiated in $2007 .{ }^{10}$ Critical care nurses and physicians receive annual training, whereas noncritical care personnel undergo biennial training. The curriculum is adaptable to institutional treatment algorithms, equipment, and code response. Content is adaptive based on provider type, unit, and opportunities for improvement as revealed by performance improvement data. Resuscitation treatment algorithms are reviewed annually by the Critical Care Committee and Code Blue Subcommittee as part of the ART program, with modifications incorporated into the institutional policies and procedures.

\section{Subjects}

All admitted patients with continuous cardiac monitoring who suffered VF/VT arrest between July 2005 and June 2013 were included in this analysis. Patients with active "do not attempt resuscitation" orders were excluded. Patients were identified from our institutional resuscitation database, into which all inhospital cardiopulmonary arrest data are entered. We did not have data on individual patient comorbidity or severity of illness. Overall patient acuity over the course of the study was monitored hospital wide through case-mix index (CMI). The index is based upon the allocation of hospital resources used to treat a diagnosis-related group of patients and has previously been used as a surrogate for patient acuity. ${ }^{11-13}$ The code $\mathrm{RN}$ who performed the resuscitation is responsible for entering data into a protected performance improvement database. Telecommunications records and the unit log are cross-referenced to assure complete capture.

\section{Protocols}

Specific protocol similarities and differences among the 3 study periods are presented in Table 1 .

\section{Stacked Shock Period (2005-2008)}

Historically, our institutional cardiopulmonary arrest protocols advocated early defibrillation with administration of 3 stacked shocks with a brief pause between each single defibrillation attempt to confirm sustained VF/VT before initiating/resuming chest compressions.

\section{Initial Chest Compression Period (2008-2011)}

In 2008 the protocol was modified to reflect recommendations to perform a 2-minute period of chest compressions prior to each defibrillation, including the initial attempt. 
TABLE 2. Demographic and Clinical Data for Study Population

\begin{tabular}{lccc}
\hline Parameter & $\begin{array}{c}\text { Stacked Shocks } \\
(\mathrm{n}=31)\end{array}$ & $\begin{array}{c}\text { Initial Chest Compressions } \\
(\mathrm{n}=33)\end{array}$ & $\begin{array}{c}\text { Modified Stack } \\
\text { Shocks ( } \mathrm{n}=42)\end{array}$ \\
\hline Age (y) & 54.3 & 64.3 & 59.8 \\
Male gender (\%) & $16(52)$ & $21(64)$ & $21(50)$ \\
VF/PVT arrest incidence (per 1,000 admissions) & 0.49 & $17(52)$ & 0.70 \\
Arrest 7 AM-5 pu (\%) & $15(48)$ & $15(45)$ & $21(50)$ \\
Non-ICU location (\%) & $13(42)$ & $31(94)$ & $17(40)$ \\
CPR prior to code team arrival (\%) & $22(71)^{*}$ & 33 & $42(100)$ \\
Perfusing rhythm after initial set of defibrillation attempts (\%) & 37 & 1.8 & $70^{\dagger}$ \\
Mean defibrillation attempts (no.) & 1.3 & $56^{\ddagger}$ & 1.5 \\
ROSC (\%) & 76 & $6(18)^{\S}$ & 90 \\
Survival-to-hospital discharge (\%) & $18(58)$ & 1.60 & $30(71)$ \\
Case-mix index & 1.51 & & $1.69 \|$ \\
(average coefficient by period) & & & \\
\hline
\end{tabular}

NOTE: Abbreviations: CPR, cardiopulmonary resuscitation; ICC, initial chest compressions; ICU, intensive care unit; MSS, modified stack shocks; PVT, pulseless ventricular tachycardia; ROSC, return of spontaneous circulation; SS, stacked shocks; VF, ventricular fibrillation. ${ }^{*} P<0.001$ versus periods SS and MSS. ${ }^{\dagger} P<0.05$ versus periods $S S$ and ICC. ${ }^{\ddagger} P<0.05$ versus period MSS. ${ }^{\S} P<0.01$ versus periods SS and MSS. $\| P<0.001$ versus periods $S S$ and ICC.

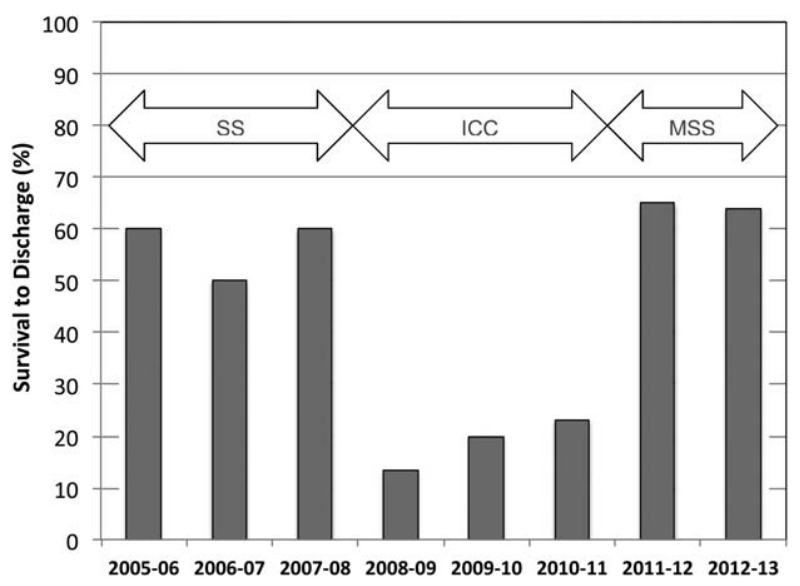

FIG. 1. Survival to discharge for patients with ventricular fibrillation/ ventricular tachycardia arrest from 2005 to 2013. Survival was significantly lower during the initial chest compression (ICC) period as compared to stacked shocks (SS) and modified stacked shock (MSS) periods $(P<0.01)$.

\section{Modified Stacked Shack Period (2011-2013)}

Finally, in 2011 the protocol was modified again, and defibrillators were configured to allow automatic advancement of defibrillation energy (120J-150J200J). The defibrillation protocol included the following elements.

For an unmonitored arrest, chest compressions and ventilations should be initiated upon recognition of cardiopulmonary arrest. If VF/VT was identified upon placement of defibrillator pads, immediate counter shock was performed and chest compressions resumed immediately for a period of 2 minutes before considering a repeat defibrillation attempt. A dose of epinephrine (1 $\mathrm{mg}$ intravenous $[\mathrm{IV}] /$ intraosseous [IO]) or vasopressin (40 units IV/IO) was administered as close to the reinitiation of chest compressions as possible. Defibrillation attempts proceeded with a single shock at a time, each preceded by 2 minutes of chest compressions.

For a monitored arrest, defibrillation attempts were expedited. Chest compressions without ventilations were initiated only until defibrillator pads were placed. Defibrillation attempts were initiated as soon as possible, with at least 3 or more successive shocks administered for persistent VF/VT (stacked shocks). Compressions were performed between shocks if they did not interfere with rhythm analysis. Compressions resumed following the initial series of stacked shocks with persistent CPA, regardless of rhythm, and pressors administered (epinephrine $1 \mathrm{mg}$ IV or vasopressin 40 units IV). Persistent VF/VT received defibrillation attempts every 2 minutes following the initial series of stacked shocks, with compressions performed continuously between attempts. Persistent VF/VT should trigger emergent cardiology consultation for possible emergent percutaneous intervention.

\section{Analysis}

The primary outcome measure was defined as survival to hospital discharge at baseline and following each protocol change. $\chi^{2}$ was used to compare the 3 time periods, with $P<0.05$ defined as statistically significant. Specific group comparisons were made with Bonferroni correction, with $P<0.017$ defined as statistically significant. Secondary outcome measures included return of spontaneous circulation (ROSC) and number of shocks required. Demographic and clinical data were also presented for each of the 3 study periods.

\section{RESULTS}

A total of 661 cardiopulmonary arrests of all rhythms were identified during the entire study period. Primary VF/VT arrests was identified in 106 patients (16\%). Of these, 102 (96\%) were being monitored with continuous ECG at the time of arrest. Demographic and clinical information for the entire study cohort are displayed in Table 2. There were no differences in age, gender, time of arrest, and location of arrest between study periods (all $P>0.05$ ). The incidence of VF/VT arrest did not vary significantly between the study periods $(P=0.16)$. There were no differences in mean 
number of defibrillation attempts per arrest; however, there was a significant improvement in the rate of perfusing rhythm after initial set of defibrillation attempts and overall ROSC favoring stacked shocks (all $P<$ 0.05 , Table 2). Survival-to-hospital discharge for all VF/VT arrest victims decreased, then increased significantly from the stacked shock period to initial chest compression period to modified stacked shock period (58\%, 18\%, 71\%, respectively, $P<0.01$, Figure 1). After Bonferroni correction, specific group differences were significant between the stacked shock and initial chest compression groups $(P<0.01)$ and modified stacked shocks and initial chest compression groups $(P<0.01$, Table 2). Finally, the incidence of "bystander CPR" appeared to be significantly greater in the modified stacked shock period following implementation of our resuscitation program (Table 2). Overall hospital CMI for fiscal years 2005/2006 through 2012/2013 were significantly different (1.47 vs $1.71, P<0.0001)$.

\section{DISCUSSION}

The specific focus of this observation was to report on defibrillation strategies that have previously only been reported in an out-of-hospital setting. There is no current consensus regarding chest compressions for a predetermined amount of time prior to defibrillation in an inpatient setting. Here we present data suggesting improved outcomes using an approach that expedited defibrillation and included a defibrillation strategy of stacked shocks (stacked shock and modified stack shock, respectively) in monitored inpatient VF/VT arrest.

Early out-of-hospital studies initially demonstrated a significant survival benefit for patients who received 1.5 to 3 minutes of chest compressions preceding defibrillation with reported arrest downtimes of 4 to 5 minutes prior to emergency medical services arrival. ${ }^{14,15}$ However, in more recent randomized controlled trials, outcome was not improved when chest compressions were performed prior to defibrillation attempt. ${ }^{16,17}$ Our findings suggest that there is no "one size fits all" approach to chest compression and defibrillation strategy. Instead, we suggest that factors including whether the arrest occurred while monitored or not aid with decision making and timing of defibrillation.

Our findings favoring expedited defibrillation and stacked shocks in witnessed arrest are consistent with the "3-phase" model of cardiac arrest proposed by Weisfeldt and Becker suggesting that defibrillation success is related to the energy status of the heart. ${ }^{18}$ In this model, the first 4 minutes of VF arrest (electrical phase) are characterized by a high-energy state with higher adenosine triphosphate (ATP)/adenosine monophosphate (AMP) ratios that are associated with increased likelihood for ROSC after defibrillation attempt. ${ }^{19}$ Further, VF appears to deplete ATP/AMP ratios after about 4 minutes, at which point the likelihood of defibrillation success is substantially diminished. ${ }^{18}$ Between 4 and 10 minutes (circulatory phase), energy stores in the myocardium are severely depleted. However, there is evidence to suggest that high-quality chest compressions and high chest compression fraction-particularly in conjunction with epinephrine-can replenish ATP stores and increase the likelihood of defibrillation success. ${ }^{6,20}$ Beyond 10 minutes (metabolic phase), survival rates are abysmal, with no therapy yet identified producing clinical utility.

The secondary analyses reveal several interesting trends. We anticipated a higher number of defibrillation attempts during phase II due to a lower likelihood of conversion with a CPR-first approach. Instead, the number of shocks was similar across all 3 periods. Our findings are consistent with previous reports of a low single or first shock probability of successful defibrillation. However, recent reports document that approximately $80 \%$ of patients who ultimately survive to discharge are successfully defibrillated within the first 3 shocks. ${ }^{21-23}$

It appears that the likelihood of conversion to a perfusing rhythm is higher with expedited, stacked shocks. This underscores the importance of identifying an optimal approach to the treatment of VF/VT, as the initial series of defibrillation attempts may determine outcomes. There also appeared to be an increase in the incidence of VF/VT during the modified stack shock period, although this was not statistically significant. The modified stack shock period correlated temporally with the expansion of our institution's cardiovascular service and the opening of a dedicated inpatient facility, which likely influenced our mixture of inpatients.

These data should be interpreted with consideration of study limitations. Primarily, we did not attempt to determine arrest times prior to initial defibrillation attempts, which is likely an important variable. However, we limited our population studied only to individuals experiencing VF/VT arrest that was witnessed by hospital care staff or occurred while on cardiac monitor. We are confident that these selective criteria resulted in expedited identification and response times well within the electrical phase. We did not evaluate differences or changes in individual patient-level severity of illness that may have potentially confounded outcome analysis. The effect of individual level in severity of illness and comorbidity are not known. Instead, we used CMI coefficients to explore hospital wide changes in patient acuity during the study period. We noticed an increasing case-mix coefficient value suggesting higher patient acuity, which would predict increased mortality rather than the decrease noted between the initial chest compression and modified stacked shock periods (Table 2). In addition, we did not integrate CPR process variables, such as depth, rate, recoil, chest compression fraction, and per-shock pauses, into this analysis. Our previous studies indicated that high-quality CPR may account 
for a significant amount of improvement in outcomes following our novel resuscitation program implementation in $2007 .{ }^{10,24}$ Since the program's inception, we have reported continuous improvement in overall inhospital mortality that was sustained throughout the duration of the study period despite the significant changes reported in the 3 periods with monitored VF/ VT arrest. ${ }^{10}$ The use of medications prior to initial defibrillation attempts was not recorded. We have recently reported that during the same period of data collection, there were no significant changes in the use of epinephrine; however, there was a significant increase in the use of vasopressin. ${ }^{10}$ It is unclear whether the increased use of vasopressin contributed to the current outcomes. However, given our cohort of witnessed in-hospital cardiac arrests with an initial shockable rhythm, we anticipate the use of vasopressors as unlikely prior to defibrillation attempt.

Additional important limitations and potential confounding factors in this study were the use of 2 different types of defibrillators, differing escalating energy strategies, and differing defibrillator waveforms. Recent evidence supports biphasic waveforms as more effective than monophasic waveforms. ${ }^{25-27}$ Comparison of defibrillator brand and waveform superiority is out the scope of this study; however, it is interesting to note similar high rates of survival in the stacked shock and modified stack shock phases despite use of different defibrillator brands and waveforms during those respective phases. Regarding escalating energy of defibrillation countershocks, the most recent 2010 AHA guidelines have no position on the superiority of either manual or automatic escalation. ${ }^{7}$ However, we noted similar high rates of survival in the stacked shock and modified stack shock periods despite use of differing escalating strategies. Finally, we used survival-tohospital discharge as our main outcome measure rather than neurological status. However, prior studies from our institution suggest that most VF/VT survivors have good neurological outcomes, which are influenced heavily by preadmission functional status. ${ }^{24}$

\section{CONCLUSIONS}

Our data suggest that in cases of monitored VF/VT arrest, expeditious defibrillation with use of stacked shocks is associated with a higher rate of ROSC and survival to hospital discharge

Disclosure: Nothing to report.

\section{References}

1. Morrison LJ, Neumar RW, Zimmerman JL, et al. Strategies for improving survival after in-hospital cardiac arrest in the United States: 2013 consensus recommendations: a consensus statement from the American Heart Association. Circulation. 2013;127:1538-1563.

2. Peberdy MA, Ornato JP, Larkin GL, et al. Survival from in-hospital cardiac arrest during nights and weekends. JAMA. 2008;299:785792.

3. Rosamond W, Flegal K, Furie K, et al. Heart disease and stroke statistics-2008 update: a report from the American Heart Association
Statistics Committee and Stroke Statistics Subcommittee. Circulation. 2008; 117:e25-e146.

4. Sasson C, Rogers MA, Dahl J, Kellermann AL. Predictors of survival from out-of-hospital cardiac arrest: a systematic review and metaanalysis. Circ Cardiovasc Qual Outcomes. 2010;3:63-81.

5. Abella BS, Alvarado JP, Myklebust H, et al. Quality of cardiopulmonary resuscitation during in-hospital cardiac arrest. JAMA. 2005;293: 305-310.

6. Christenson J, Andrusiek D, Everson-Stewart S, et al. Chest compression fraction determines survival in patients with out-of-hospital ventricular fibrillation. Circulation. 2009;120:1241-1247.

7. Jacobs I, Sunde K, Deakin CD, et al. Part 6: Defibrillation: 2010 International Consensus on Cardiopulmonary Resuscitation and Emergency Cardiovascular Care Science With Treatment Recommendations. Circulation. 2010;122:S325-S337.

8. Field JM, Hazinski MF, Sayre MR, et al. Part 1: executive summary: 2010 American Heart Association Guidelines for Cardiopulmonary Resuscitation and Emergency Cardiovascular Care. Circulation. 2010; 122:S640-S656.

9. Neumar RW, Otto CW, Link MS, et al. Part 8: adult advanced cardiovascular life support: 2010 American Heart Association Guidelines for Cardiopulmonary Resuscitation and Emergency Cardiovascular Care. Circulation. 2010;122:S729-S767.

10. Davis DP, Graham PG, Husa RD, et al. A performance improvementbased resuscitation programme reduces arrest incidence and increases survival from in-hospital cardiac arrest. Resuscitation. 2015;92: 63-69.

11. Averill RF. The evolution of case-mix measurement using DRGs: past, present and future. Stud Health Technol Inform. 1994;14:75-83.

12. Merchant RM, Yang L, Becker LB, et al. Variability in case-mix adjusted in-hospital cardiac arrest rates. Med Care. 2012;50:124-130.

13. Timbie JW, Hussey PS, Adams JL, Ruder TW, Mehrotra A. Impact of socioeconomic adjustment on physicians' relative cost of care. Med Care. 2013;51:454-460.

14. Cobb LA, Fahrenbruch CE, Walsh TR, et al. Influence of cardiopulmonary resuscitation prior to defibrillation in patients with out-ofhospital ventricular fibrillation. JAMA. 1999;281:1182-1188.

15. Wik L, Hansen TB, Fylling F, et al. Delaying defibrillation to give basic cardiopulmonary resuscitation to patients with out-of-hospital ventricular fibrillation: a randomized trial. JAMA. 2003;289:13891395.

16. Baker PW, Conway J, Cotton C, et al. Defibrillation or cardiopulmonary resuscitation first for patients with out-of-hospital cardiac arrests found by paramedics to be in ventricular fibrillation? A randomised control trial. Resuscitation. 2008;79:424-431.

17. Jacobs IG, Finn JC, Oxer HF, Jelinek GA. CPR before defibrillation in out-of-hospital cardiac arrest: a randomized trial. Emerg Med Australas. 2005;17:39-45.

18. Weisfeldt ML, Becker LB. Resuscitation after cardiac arrest: a 3-phase time-sensitive model. JAMA. 2002;288:3035-3038.

19. Salcido DD, Menegazzi JJ, Suffoletto BP, Logue ES, Sherman LD. Association of intramyocardial high energy phosphate concentrations with quantitative measures of the ventricular fibrillation electrocardiogram waveform. Resuscitation. 2009;80:946-950.

20. Holzer M, Behringer W, Sterz F, et al. Ventricular fibrillation median frequency may not be useful for monitoring during cardiac arrest treated with endothelin-1 or epinephrine. Anesth Analg. 2004;99: 1787-1793, table of contents.

21. Eftestøl T, Sunde K, Aase SO, Husøy JH, Steen PA. "Probability of successful defibrillation" as a monitor during CPR in out-of-hospital cardiac arrested patients. Resuscitation. 2015;48:245-254.

22. Rodriguez-Nunez A, Lopez-Herce J, Del Castillo J, Bellon JM. Shockable rhythms and defibrillation during in-hospital pediatric cardiac arrest. Resuscitation. 2014;85:387-391.

23. Tomkins WG, Swain AH, Bailey M, Larsen PD. Beyond the pre-shock pause: the effect of prehospital defibrillation mode on CPR interruptions and return of spontaneous circulation. Resuscitation. 2013;84: 575-579.

24. Sell RE, Lawrence B, Davis DP. Implementing a "resuscitation bundle" decreases incidence and improves outcomes in inpatient cardiopulmonary arrest. Circulation 2009;120(18 Suppl):S1441.

25. Schneider T, Martens PR, Paschen H, et al. Multicenter, randomized, controlled trial of 150-J biphasic shocks compared with 200- to 360-J monophasic shocks in the resuscitation of out-of-hospital cardiac arrest victims. Optimized Response to Cardiac Arrest (ORCA) Investigators. Circulation. 2000;102:1780-1787.

26. van Alem AP, Chapman FW, Lank P, Hart AA, Koster RW. A prospective, randomised and blinded comparison of first shock success of monophasic and biphasic waveforms in out-of-hospital cardiac arrest. Resuscitation. 2003;58:17-24.

27. Morrison LJ, Dorian P, Long J, et al. Out-of-hospital cardiac arrest rectilinear biphasic to monophasic damped sine defibrillation waveforms with advanced life support intervention trial (ORBIT). Resuscitation. 2005;66:149-157. 